Structural Eng. / Earthquake Eng. Vol.4. No.1, 51 s-59s. April 1987

Japan Society of Civil Engineers (Proc. of JSCE No.380/I-7)

\title{
BENDING ANALYSIS OF RECTANGULAR PLATE ON NON-UNIFORM ELASTIC FOUNDATIONS
}

\author{
By Hiroshi MATSUDA* and Takeshi SAKIYAMA**
}

\begin{abstract}
In this paper, an approximate method for analyzing the bending problems of rectangular Mindlin plates on elastic foundations is proposed. The solutions of the partial differential equations of the bending are obtained in the discrete form, by translating the differential equations into integral equations and applying numerical integration.

In order to confirm the convergency and accuracy of numerical solutions, comparisons with numerical solutions obtained by other investigators are made. As the application, the bending behavior of rectangular plate on nonlinear elastic foundations and on non-uniform elastic foundations are calculated.

Keywords : rectangular plate, nonlinear elastic foundations, non-uniform elastic foundations
\end{abstract}

\section{INTRODUCTION}

The problems on the bending of rectangular plate on elastic foundations have been analyzed by many investigators, by using numerical methods such as FEM, FDM and series solutions.

Nohmachil) expanded "Finite Fourier Transformation" to biharmonic differential equation by means of Green's formula, and gave the solutions for the bending of the rectangular plate on elastic foundations with four free edges. Kurata, Takahashi and Tanihira ${ }^{2)}$ obtained the numerical solutions of rectangular plate with all free boundaries on nonlinear elastic foundations, using approximate method applying the finite difference method. Kitamura and Sakurai ${ }^{3}$ analyzed rectangular plates with four edges free on elastic foundations, using the method of eigenfunction expansion.

Cheung and Zienkiewicz ${ }^{4}$ first analyzed the plate on elastic foundations, applying finite element method. Henry $^{5)}$ analyzed the large deflection problems of the rectangular plate on elastic foundations by FEM. $\mathrm{Svec}^{6)}$ analyzed the thick plate on elastic foundations by FEM.

Sonoda and Kobayashi ${ }^{7,8)}$ analyzed the quasistatic bending of rectangular plates resting on linear viscoelastic foundations obeying Winkler's hypothesis, and obtained double series solutions which are derived by means of eigenfunction expansions.

There are many methods to analyze the bending of rectangular plate on elastic foundations. However, it has been hardly carried out to study the bending of rectangular plates with variable thickness on non-uniform elastic foundations. And since the fundamental differential equations are formed by the simultaneous partial differential equations with variable coefficients which are composed of the flexural rigidity of the plate $D(x, y)$, the thickness of the plate $h(x, y)$ and foundation modulus $k(x, y)$, it is

* Member of JSCE, M. Eng., Research Associate, University of Nagasaki (Nagasaki city, Nagasaki).

** Member of JSCE, Dr. Eng., Associate Professor, University of Nagasaki (Nagasaki city, Nagasaki). 
therefore very difficult to find the exact solution.

In this paper, an approximate method for analyzing the bending problems of rectangular Mindlin plates with variable thickness resting on elastic foundations which foundation moduli are locally non-uniform has been proposed. By translating the differential equations into integral equations and applying numerical integration, the solutions of the differential equations are obtained in the discrete form, and give the transverse shear forces, the twisting moments, the bending moments, the rotations and the deflections at the all discrete points which are intersections of the vertical and horizontal equally dividing lines on the plate.

In order to confirm the convergency and accuracy of the numerical solutions obtained by the present method, comparisons with the exact solutions obtained by other investigators are made. As the application of the present method, the bending behavior of the rectangular plate on nonlinear elastic foundations and on non-uniform elastic foundations are calculated.

Furthermore, it is possible to analyze the rectangular plate with arbitrary boundary conditions, load conditions and plate thickness resting on non-uniform elastic foundations, by using the present method.

\section{FUNDAMENTAL DIFFERENTIAL EQUATIONS FOR ELASTIC FOUNDATIONS PROBLEM}

The fundamental differential equations for the bending of rectangular plate with variable thickness on elastic foundations as shown in Fig. 1 are the simultaneous partial differential equations $(1 \cdot a) \sim(1 \cdot h)$ as follows. These equations are based on Mindlin's theory which include the effect of shear deformation. Here, the shear coefficient $x$ is taken equal to 5/6.

$$
\begin{aligned}
& \frac{\partial Q_{x}}{\partial x}+\frac{\partial Q_{y}}{\partial y}+q-k_{v} w=0 \\
& \frac{\partial M_{x}}{\partial x}+\frac{\partial M_{x y}}{\partial y}-Q_{x}=0 \cdots \cdots \\
& \frac{\partial M_{y}}{\partial y}+\frac{\partial M_{x y}}{\partial x}-Q_{y}=0 \cdots \cdots \\
& \frac{\partial \theta_{y}}{\partial y}+\nu \frac{\partial \theta_{x}}{\partial x}=\frac{M_{y}}{D} \cdots \ldots \ldots . .
\end{aligned}
$$

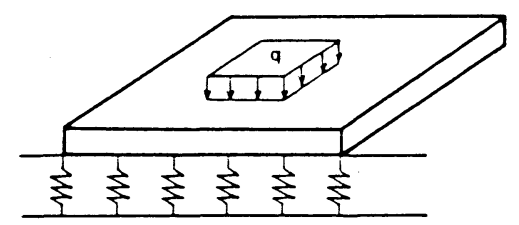

Fig. 1 Rectangular plate on elastic foundations.

Here, $Q_{y}, Q_{x}$ are the shearing forces, $M_{x y}$ is the torsional moment, $M_{y}$ and $M_{x}$ are the bending moments, $\theta_{y}$ and $\theta_{x}$ are the slopes, $w$ is the deflection, $q=q(x, y)$ is the lateral load intensity, $E$ is the modulus of elasticity, $G$ is the shear modulus of elasticity, $\nu$ is Poisson's ratio, $h=h(x, y)$ is the plate thickness, $D$ $=E h^{3} / 12\left(1-\nu^{2}\right)$ is the flexural rigidity of the plate, $k_{v}=k_{v}(x, y)$ is the elastic modulus of the foundation.

By using the non-dimensional expression, $Q_{y}, Q_{x}, M_{x y}, M_{y}, M_{x}, \theta_{y}, \theta_{x}$ and $w$ are as follows:

$$
\left(X_{1}, X_{2}\right)=\frac{a^{2}}{D_{0}\left(1-\nu^{2}\right)}\left(Q_{y}, Q_{x}\right), \quad\left(X_{3}, X_{4}, X_{5}\right)=\frac{a}{D_{0}\left(1-\nu^{2}\right)}\left(M_{x y}, M_{y}, M_{x}\right), \quad\left(X_{6}, X_{7}\right)=\left(\theta_{y}, \theta_{x}\right), \quad X_{8}=\frac{w}{a}
$$

the differential equations $(1 \cdot a) \sim(1 \cdot h)$ are rewritten as follows;

$$
\begin{aligned}
& \frac{\partial X_{1}}{\partial \zeta}+\mu \frac{\partial X_{2}}{\partial \eta}=-\bar{q}+\bar{k}_{v} X_{8} \\
& \frac{\partial X_{3}}{\partial \zeta}+\mu \frac{\partial X_{5}}{\partial \eta}=\mu X_{2} \cdots \cdots \cdots \\
& \frac{\partial X_{4}}{\partial \zeta}+\mu \frac{\partial X_{3}}{\partial \eta}=\mu X_{1} \cdots \cdots \cdots \\
& \frac{\partial X_{6}}{\partial \zeta}+\nu \mu \frac{\partial X_{7}}{\partial \eta}=I X_{4} \cdots \cdots \cdots
\end{aligned}
$$$$
\begin{aligned}
& \nu \frac{\partial X_{6}}{\partial \zeta}+\mu \frac{\partial X_{7}}{\partial \eta}=I X_{5} \\
& \frac{\partial X_{7}}{\partial \zeta}+\mu \frac{\partial X_{6}}{\partial \eta}=J X_{3} \cdots \\
& \frac{\partial X_{8}}{\partial \eta}+X_{7}=L X_{2} \cdots \cdots \\
& \frac{\partial X_{8}}{\partial \zeta}+\mu X_{6}=\mu L X_{1} \cdots
\end{aligned}
$$ 
Here, $x=a \eta, y=b \zeta, a, b$ : side length of rectangular plate, $h_{0}$ is the standard plate thickness, $q_{0}$ is the standard load intensity, $\mu=b / a, k_{v 0}$ is the standard elastic modulus of the foundation, $D_{0}$ is the standard flexural rigidity of the plate, $D_{0}=E h_{0}^{3} / 12\left(1-\nu^{2}\right)$

$$
\begin{aligned}
& \bar{q}=\mu K_{1} \frac{q}{q_{0}}, \quad K_{1}=\frac{q_{0} a^{3}}{D_{0}\left(1-\nu^{2}\right)}, \quad \bar{k}_{v}=\mu K_{2} \frac{k_{v}}{k_{v 0}}, \quad K_{2}=\frac{k_{v 0} a^{4}}{D_{0}\left(1-\nu^{2}\right)} \\
& I=\mu\left(1-\nu^{2}\right)\left(\frac{h_{0}}{h}\right)^{3}, \quad J=2 \mu(1+\nu)\left(\frac{h_{0}}{h}\right)^{3}, \quad L=\frac{1}{10} \frac{E}{G}\left(\frac{h_{0}}{a}\right)^{2} \frac{h_{0}}{h}
\end{aligned}
$$

The differential equations $(2 \cdot a) \sim(2 \cdot h)$ can be used to analyzed the bending of rectangular plate with variable thickness on non-uniform elastic foundations with arbitrary boundary conditions and arbitrary load conditions.

\section{APPROXIMATE SOLUTION OF DIFFERENTIAL EQUATIONS}

It is impossible to obtain the exact solutions of differential equations $(2 \cdot a) \sim(2 \cdot h)$ under the arbitrary loading and boundary conditions, since these equations are formed by simultaneous partial differential equations with variable coefficients. Therefore, in this paper, we consider the rectangular plate as assembly of intersections as shown in Fig. 2. Approximate solutions of differential equations are obtained for these discrete points.

One divides the bounded area $[0, a],[0, b]$ into $m, n$ equal-interval, and numbers each divisional point, as shown in Fig. 2. Now, let $[i, j]$ be any point in the rectangular region $0 \leqq \eta \leqq \eta_{i}, 0 \leqq \zeta \leqq \zeta_{j}$, $\bigcirc$ be the principal point, $\bigcirc$ be the interior subordinate points, and $\bigcirc$ be the boundary subordinate points.

First, by integrating the differential equations $(2 \cdot a) \sim(2 \cdot h)$

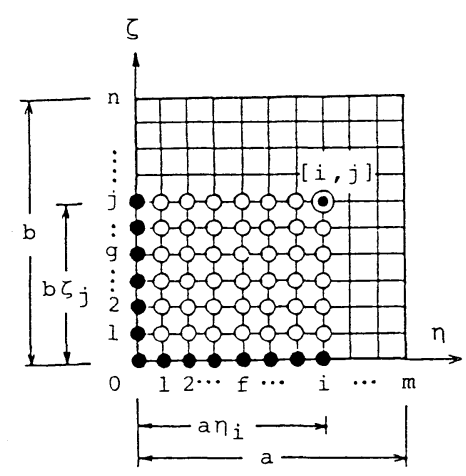

Fig. 2 Discrete points on rectangular plate. over the area $\left[0, \eta_{i}\right]$ and $\left[0, \zeta_{j}\right]$, one obtains the integral equations. Next, by applying the numerical integral, one can obtain the simultaneous equations for nondimension values $X_{p i j}$ of the principal point. By solving these equations, one can obtain eq. (3). The details to obtain eq. (3) see Ref. (9).

$$
\begin{aligned}
X_{p i j}= & \sum_{t=1}^{8}\left\{\sum_{f=0}^{i} \beta_{i f} A_{p t}\left[X_{t f 0}-X_{t f j}\left(1-\delta_{f i}\right)\right]+\sum_{g=0}^{j} \beta_{j g} B_{p t}\left[X_{t 0 g}-X_{t i g}\left(1-\delta_{g j}\right)\right]\right. \\
& \left.+\sum_{f=0}^{i} \sum_{g=0}^{j} \beta_{i f f} \beta_{j g} C_{p t f g} X_{t f g}\left(1-\delta_{f i} \delta_{g j}\right)\right\}-\sum_{f=0}^{i} \sum_{g=0}^{j} \beta_{i f f} \beta_{j g} A_{p 1} \bar{q}_{f g} \ldots \ldots \ldots \ldots
\end{aligned}
$$

where $p=1,2, \cdots, 8, \quad i=1,2, \cdots, m, \quad j=1,2, \cdots, n, \quad \beta_{i f}=\alpha_{i s} / m, \quad \beta_{j g}=\alpha_{j g} / n . \quad A_{p t}, B_{p t}, C_{p t f g}$ : Appendix, $\delta_{i j}:$ Kronecker's delta

The coefficients $\alpha_{i f}, \alpha_{j g}$ are the weight coefficients of the numerical integration. The trapezoidal rule of approximate numerical integration are applied in this paper, therefore the values of $\alpha_{i f}, \alpha_{j g}$ are given as follows : $\alpha_{i f}=1-\left(\delta_{0 f}+\delta_{i f}\right) / 2, \quad \alpha_{j g}=1-\left(\delta_{0 g}+\delta_{j g}\right) / 2$

As one calculates the values $X_{p 11}, X_{p 12}, X_{p 21}, \cdots$, in numbered order by using eq. ( 3 ), the values of the all interior subordinate points are eliminated. Ultimately, the values $X_{\rho i j}$ of the principal point are represented by the values $X_{r f o}(r=1,3,4,6,7,8), X_{s 0 g}(s=2,3,5,6,7,8)$ of the bounded subordinate points as follows;

$$
X_{p i j}=\sum_{d=1}^{6}\left(\sum_{f=0}^{i} a_{1 p i j f d} \cdot X_{r f 0}+\sum_{g=0}^{j} a_{2 p i j g d} \cdot X_{s 0 g}\right)+q_{p i j}
$$

where,

$$
\begin{aligned}
a_{h p i j u d}= & \sum_{t=1}^{8}\left\{\sum_{k=0}^{i} \beta_{i k} A_{p t L}\left[a_{h t k 0 u d}-a_{h t k j u d}\left(1-\delta_{k i}\right)\right]+\sum_{l=0}^{j} \beta_{j l} B_{p t}\left[a_{h t o l u d}-a_{h t i l u d}\left(1-\delta_{l j}\right)\right]\right. \\
& \left.+\sum_{k=0}^{i} \sum_{l=0}^{j} \beta_{i \hbar} \beta_{j l} C_{p t k l} a_{h t k l u d}\left(1-\delta_{k i} \delta_{l j}\right)\right\}
\end{aligned}
$$




$$
\begin{aligned}
& q_{p i j}=\sum_{i=1}^{8}\left\{\sum_{k=0}^{i} \beta_{i k} A_{p t}\left[q_{t k 0}-q_{t k j}\left(1-\delta_{k i}\right)\right]+\sum_{l=0}^{j} \beta_{j l} B_{p t}\left[q_{t 0 l}-q_{t i l}\left(1-\delta_{l j}\right)\right]\right. \\
& \left.\quad+\sum_{k=0}^{i} \sum_{l=0}^{j} \beta_{i k} \beta_{j l} C_{p t k l} q_{t k l}\left(1-\delta_{k i} \delta_{l j}\right)\right\}-\sum_{k=0}^{i} \sum_{l=0}^{j} \beta_{i k} \beta_{j l} A_{p 1} \bar{q}_{k l} \\
& p=1 \sim 8, \quad i=1 \sim m, \quad f=0 \sim i, \quad g=0 \sim j, \quad d=1 \sim 6, \quad h=1,2, \quad u=f(h=1) \quad \text { or } g(h=2) \\
& a_{1 r i 0 i d}=1, \quad a_{2 s 0 j j d}=1 \\
& a_{110 j 01}=\frac{x_{00}}{x_{0 j}} \bar{\alpha}_{j}, \quad a_{22 i 001}=\frac{x_{00}}{x_{i 0}} \bar{\alpha}_{i}, \quad a_{140 j 03}=\frac{\bar{D}_{00}}{\bar{D}_{0 j}} \bar{\alpha}_{j}, \quad a_{25 i 003}=\frac{\bar{D}_{00}}{\bar{D}_{i 0}} \bar{\alpha}_{i} \\
& a_{12 i 0 k 5}=\frac{1}{x_{i 0}} \bar{\beta}_{i k}, \quad a_{210 j l 4}=\frac{1}{x_{0 j}} \bar{\beta}_{j l}, \quad a_{15 i 0 k 3}=\nu \frac{\bar{D}_{00}}{\bar{D}_{i 0}} \bar{\beta}_{i k}, \quad a_{240 j l 3}=\nu \frac{\bar{D}_{0 l}}{\bar{D}_{0 j}} \bar{\beta}_{j l} \\
& a_{12 i 0 k 5}=\frac{1}{x_{i 0}} \bar{\gamma}_{i k}, \quad a_{210 j l 6}=\frac{1}{\mu x_{0 j}} \bar{\gamma}_{j l}, \quad a_{15 i 0 k 5}=\frac{1}{\bar{D}_{i 0}} \bar{\gamma}_{i k}, \quad a_{240 j l 4}=\frac{1}{\mu D_{0 j}} \bar{\gamma}_{j l} \\
& \bar{\alpha}_{i}=(-1)^{i}, \quad \bar{\beta}_{i k}=\delta_{i k}+(-1)^{i+1} \cdot \delta_{0 k}, \quad \bar{\gamma}_{i k}=\frac{4 m(-1)^{i+k}}{1+\delta_{i k}+\delta_{0 k}}, \quad \bar{D}_{i 0}=\frac{D_{0}}{D_{i 0}} \\
& s=d+\delta_{1 d}+\delta_{2 d}+2\left(\delta_{3 d}+\delta_{4 d}+\delta_{5 d}+\delta_{6 d}\right), \quad r=d+\delta_{2 d}+\delta_{3 d}+2\left(\delta_{4 d}+\delta_{5 d}+\delta_{6 d}\right)
\end{aligned}
$$

Eq. (4) is an approximate solution of the differential equations $(2 \cdot \mathrm{a}) \sim(2 \cdot \mathrm{h})$. The values $X_{r f 0}, X_{s 0 g}$ of the bounded subordinate points are integral constants. Then, the coefficients $a_{\text {hijud }}$ which relate the values $X_{p i j}$ with the value $X_{r s 0}, X_{s 0 g}$ correspond to the transfer matrix of the Transfer Matrix Method.

\section{INTEGRAL CONSTANTS AND BOUNDARY CONDITIONS}

Integral constants $X_{r f 0}$ and $X_{s 0 g}$ express dimensionless quantities with respect to $Q_{y}, M_{x y}, M_{y}, \theta_{y}, \theta_{x}, w$ and $Q_{x}, M_{x y}, M_{x}, \theta_{y}, \theta_{x}, w$, on $\zeta=0$ and $\eta=0$, respectively.

There are six integral constants on each discrete point. According to the support type of the bounded sides, three integral constants of six are known, and the remaining three unknown integral constants are determined by the boundary conditions along $\eta=1.0$ and $\zeta=1.0$.

Integral constants and boundary conditions of rectangular plate with four edges free and with two opposite edges simply supported and the other two edges free are shown in Fig. 3 and Fig. 4, respectively. In these figures, fig. (a) express the whole of a rectangular plate, fig. (c) and fig. (d) express one half of a rectangular plate with one symmetrical axis (either right and left or up and down), and fig. (b) represent

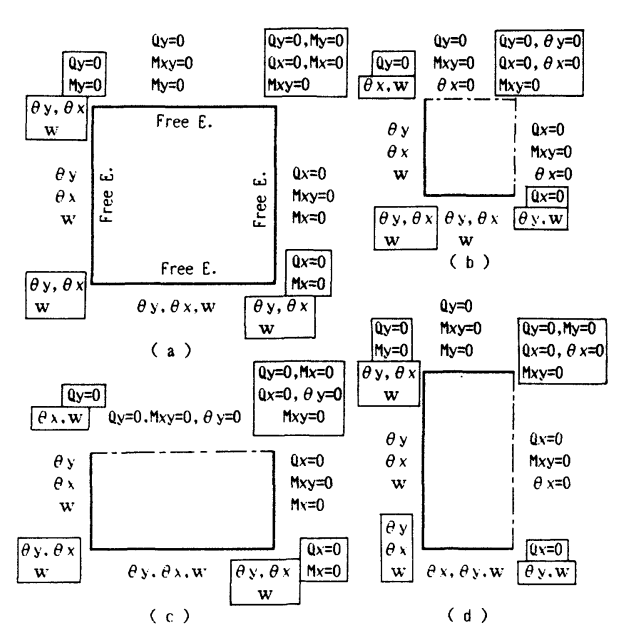

Fig. 3 Integral constants and boundary conditions of plate with four free edges.
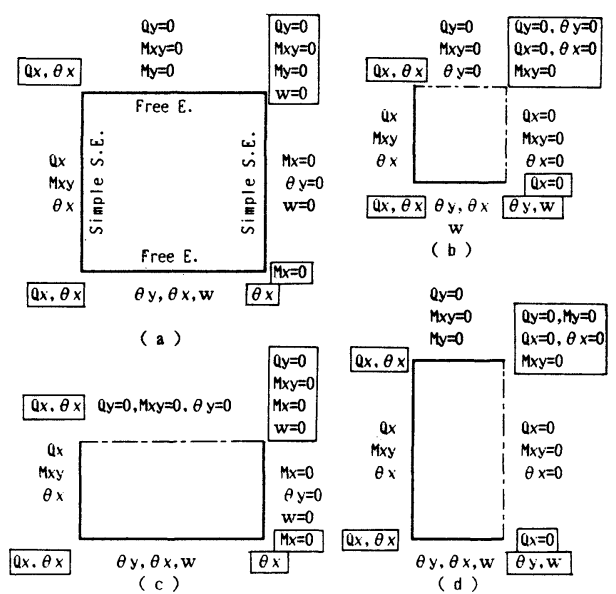

Fig. 4 Integral constants and boundary conditions of plate with two opposite edges simply supported and the other two free. 
one quater of a rectangular plate with two symmetrical axes. Integral constants and boundary conditions at the corners of plate are shown in the boxes.

\section{NUMERICAL RESULTS}

\section{(1) Rectangular plate on uniform linear elastic foundations}

First, in order to investigate the convergency and accuracy of numerical solutions of the rectangular plate on uniform linear elastic foundations, let the present method be applied to a square plate with two opposite edges simply supported and the other two free, which is subjected to a uniform distributed load $q$ over the square area in the center of plate as shown in Fig. 5. Table 1 shows the numerical results of the deflections and the bending moments at the center and at the middle point of free edge of the plate with different $h / a$ values, together with the series solutions obtained by Ref. ( 8 ). In this table, $m=10$ and 20 are division number, and 10-20 represents the Richardson's extrapolated values of $m=10,20$. It is found from this table that the extrapolated values (10-20) agree with the series solutions.

Second, let the present method be applied to a square plate with four free edges, which is subjected to a uniform distributed load $q$ over the square area in the center of plate as shown in Fig. 5, and a concentrated load $P$ at the center, respectively. The values of the deflections, the bending moments and reactions at the center, at the corner and at the middle point of free edge of the plate are shown in Table 2 and Table 3. In these tables, $10-20,8-12$ and $4-8-12$ repesent the Richardson's extrapolated values. It is found from

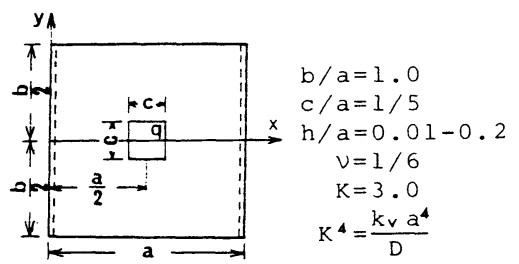

Fig. 5 Partially loaded square plate on elastic foundations.

Table 1 Deflections and bending moments of the rectangular plate with two opposite edges simply supported and the other two free subjected to uniform distributed load $q(\nu=1 / 6, \quad b / a=1.0, \quad c / a=1 / 5, \quad K=3.0)$.

\begin{tabular}{|c|c|c|c|c|c|c|}
\hline \multirow{2}{*}{$\mathrm{h} / \mathrm{a}$} & \multirow{2}{*}{ m } & \multicolumn{3}{|c|}{$x=a / 2, y=0$} & \multicolumn{2}{|c|}{$x=a / 2, y=b / 2$} \\
\hline & & w & $M x$ & My & w & $M x$ \\
\hline 0.01 & $\begin{array}{c}10 \\
20 \\
10-20 \\
\text { Ref. } 8\end{array}$ & $\begin{array}{l}0.504 \\
0.526 \\
0.534 \\
0.535\end{array}$ & $\begin{array}{l}0.707 \\
0.798 \\
0.828 \\
0.824\end{array}$ & $\begin{array}{l}0.448 \\
0.533 \\
0.562 \\
0.560\end{array}$ & $\begin{array}{l}0.345 \\
0.347 \\
0.348 \\
0.349\end{array}$ & $\begin{array}{l}0.348 \\
0.349 \\
0.350 \\
0.349\end{array}$ \\
\hline 0.05 & $\begin{array}{c}10 \\
20 \\
10-20 \\
\text { Ref.8 }\end{array}$ & $\begin{array}{l}0.508 \\
0.532 \\
0.540 \\
0.541\end{array}$ & $\begin{array}{l}0.708 \\
0.797 \\
0.826 \\
0.824\end{array}$ & $\begin{array}{l}0.447 \\
0.534 \\
0.564 \\
0.562\end{array}$ & $\begin{array}{l}0.343 \\
0.346 \\
0.346 \\
0.346\end{array}$ & $\begin{array}{l}0.351 \\
0.354 \\
0.355 \\
0.355\end{array}$ \\
\hline 0.10 & $\begin{array}{c}10 \\
20 \\
10-20 \\
\text { Ref.8 }\end{array}$ & $\begin{array}{l}0.522 \\
0.549 \\
0.558 \\
0.558\end{array}$ & $\begin{array}{l}0.705 \\
0.796 \\
0.826 \\
0.820\end{array}$ & $\begin{array}{l}0.447 \\
0.536 \\
0.565 \\
0.562\end{array}$ & $\begin{array}{l}0.342 \\
0.344 \\
0.344 \\
0.344\end{array}$ & $\begin{array}{l}0.355 \\
0.360 \\
0.361 \\
0.359\end{array}$ \\
\hline 0.15 & $\begin{array}{c}10 \\
20 \\
10-20 \\
\text { Ref. } 8\end{array}$ & $\begin{array}{l}0.545 \\
0.576 \\
0.586 \\
0.587\end{array}$ & $\begin{array}{l}0.699 \\
0.786 \\
0.815 \\
0.813\end{array}$ & $\begin{array}{l}0.447 \\
0.535 \\
0.564 \\
0.562\end{array}$ & $\begin{array}{l}0.340 \\
0.342 \\
0.343 \\
0.343\end{array}$ & $\begin{array}{l}0.356 \\
0.360 \\
0.361 \\
0.361\end{array}$ \\
\hline 0.20 & $\begin{array}{c}10 \\
20 \\
10-20 \\
\text { Ref.8 }\end{array}$ & $\begin{array}{l}0.577 \\
0.613 \\
0.625 \\
0.626\end{array}$ & $\begin{array}{l}0.690 \\
0.777 \\
0.806 \\
0.804\end{array}$ & $\begin{array}{l}0.447 \\
0.535 \\
0.564 \\
0.562\end{array}$ & $\begin{array}{l}0.339 \\
0.340 \\
0.341 \\
0.341\end{array}$ & $\begin{array}{l}0.356 \\
0.359 \\
0.361 \\
0.361\end{array}$ \\
\hline Mul & lier & $10^{-3} \mathrm{qa}^{4} / \mathrm{D}$ & & & $10^{-3} \mathrm{qa}^{4} / \mathrm{D}$ & $10^{-2} \mathrm{qa}^{2}$ \\
\hline
\end{tabular}
Table 3 that the numerical solutions obtained by the present method converged monotonically according to an increase of division number.

(2) Variable thickness plate on nonlinear elastic foundations

Let the present method be applied to a square plate of variable thickness with four free edges on nonlinear tensionless elastic foundations, which is subjected to a concentrated load $P$ at the center.

Nonlinear tensionless elastic foundations are assumed to be active only when the plate has pressed against the foundations, and to be inactive in the regions where the plate has lifted off of the foundations. In other words, the foundations can be modelled as the springs of the stiffness $k$ when in compression and zero when in tension. In this paper, the load-deflection relationship of the nonlinear elastic foundation is assumed as shown in Fig. 6 , and it is expressed as follows.

$$
p=k \bar{w} w /(\bar{w}+w)
$$

The modified spring reaction $p^{\prime}$ at $w=w^{\prime}$ is

$$
p^{\prime}=k \bar{w}^{2} w /\left(\bar{w}+w^{\prime}\right)^{2}+k \bar{w} w^{2} /\left(\bar{w}+w^{\prime}\right)^{2}
$$

Fig. 7 shows the flow-chart of computational procedures. Now, if the $(s-1)$ th deflection at the point 
Table 2 Deflections and bending moments of the rectangular plate with four free edges subjected to uniform distributed load $q(\nu=1 / 6, \quad b / a=1.0, \quad c / a=1 / 5, \quad K=3.0)$.

\begin{tabular}{|c|c|c|c|c|c|c|c|c|}
\hline \multirow{2}{*}{$\mathrm{h} / \mathrm{a}$} & \multirow{2}{*}{$\mathrm{m}$} & \multicolumn{3}{|c|}{$(\mathrm{a} / 2,0)$} & $(0, \mathrm{~b} / 2)$ & $(\mathrm{a} / 2, \mathrm{~b} / 2)$ \\
\cline { 3 - 9 } & & $w$ & $M x$ & $p$ & $p$ & $w$ & $w$ & $M x$ \\
\hline \multirow{3}{*}{0.01} & 10 & 0.687 & 0.504 & 5.56 & 2.13 & 0.263 & 0.428 & 0.170 \\
& 20 & 0.708 & 0.596 & 5.73 & 2.01 & 0.248 & 0.424 & 0.187 \\
& $10-20$ & 0.714 & 0.627 & - & - & 0.243 & 0.422 & 0.193 \\
\hline \multirow{3}{*}{0.10} & 10 & 0.702 & 0.501 & 5.69 & 2.12 & 0.262 & 0.425 & 0.182 \\
& 20 & 0.726 & 0.597 & 5.88 & 2.00 & 0.247 & 0.420 & 0.354 \\
& $10-20$ & 0.734 & 0.630 & - & - & 0.242 & 0.419 & 0.355 \\
\hline \multirow{2}{*}{0.20} & 10 & 0.744 & 0.496 & 6.03 & 2.07 & 0.255 & 0.418 & 0.186 \\
& 20 & 0.779 & 0.589 & 6.31 & 1.94 & 0.240 & 0.413 & 0.197 \\
& $10-20$ & 0.790 & 0.620 & - & - & 0.236 & 0.411 & 0.200 \\
\hline \multicolumn{2}{|l|}{ Multiplier } & $10^{-3} \mathrm{qa}^{4} / \mathrm{D}$ & $10^{-2} \mathrm{qa}^{2}$ & \multicolumn{7}{|c|}{$10^{-2} \mathrm{q}$} & $10^{-3} \mathrm{qa}^{4} / \mathrm{D}$ & $10^{-2} \mathrm{qa}^{2}$ \\
\hline
\end{tabular}

Table 3 Deflections and bending moments of the rectangular plate with four free edges subjected to a concentrated load $P$ at the center $(\nu=1 / 6, \quad b / a=1.0, \quad K=3.0)$.

\begin{tabular}{|c|c|c|c|c|c|c|c|}
\hline \multirow{2}{*}{$\mathrm{h} / \mathrm{a}$} & \multirow{3}{*}{$\mathrm{m}$} & \multicolumn{3}{|c|}{$(\mathrm{a} / 2,0)$} & \multicolumn{2}{c|}{$(0, \mathrm{~b} / 2)$} & $\mathrm{a} / 2, \mathrm{~b} / 2$ \\
\cline { 3 - 8 } & & $\mathrm{w}$ & $\mathrm{Mx}$ & $\mathrm{p}$ & $\mathrm{w}$ & $\mathrm{p}$ & $\mathrm{w}$ \\
\hline \multirow{5}{*}{0.01} & 4 & 0.0158 & 0.085 & 1.28 & 0.00817 & 0.66 & 0.0112 \\
& 8 & 0.0175 & 0.148 & 1.42 & 0.00641 & 0.52 & 0.0106 \\
& 12 & 0.0179 & 0.185 & 1.45 & 0.00608 & 0.49 & 0.0105 \\
& 16 & 0.0182 & 0.211 & 1.47 & 0.00596 & 0.48 & 0.0105 \\
& $8-12$ & 0.0183 & 0.214 & - & 0.00581 & - & 0.0104 \\
& $4-8-12$ & 0.0184 & 0.220 & - & 0.00581 & - & 0.0104 \\
\hline \multirow{5}{*}{0.10} & 4 & 0.0160 & 0.085 & 1.30 & 0.00812 & 0.66 & 0.0111 \\
& 8 & 0.0179 & 0.147 & 1.45 & 0.00638 & 0.52 & 0.0105 \\
& 12 & 0.0185 & 0.185 & 1.50 & 0.00608 & 0.49 & 0.0105 \\
& $8-12$ & 0.0189 & 0.211 & 1.53 & 0.00596 & 0.48 & 0.0105 \\
& $4-8-12$ & 0.0190 & 0.213 & - & 0.00580 & - & 0.0104 \\
\hline \multirow{5}{*}{0.20} & 4 & 0.0166 & 0.084 & 1.34 & 0.00797 & 0.65 & 0.0109 \\
& 8 & 0.0192 & 0.145 & 1.56 & 0.00622 & 0.50 & 0.0103 \\
& 12 & 0.0203 & 0.182 & 1.64 & 0.00589 & 0.48 & 0.0102 \\
& 16 & 0.0209 & 0.209 & 1.69 & 0.00578 & 0.47 & 0.0102 \\
& $8-12$ & 0.0211 & 0.212 & - & 0.00562 & - & 0.0102 \\
& $4-8-12$ & 0.0213 & 0.218 & - & 0.00562 & - & 0.0102 \\
\hline \multicolumn{2}{|l|}{ Multiplier } & $\mathrm{Pa}^{2} / \mathrm{D}$ & $\mathrm{P}$ & $\mathrm{P} / \mathrm{a}^{2}$ & $\mathrm{~Pa}^{2} / \mathrm{D}$ & $\mathrm{P} / \mathrm{a}^{2}$ & $\mathrm{~Pa}^{2} / \mathrm{D}$ \\
\hline
\end{tabular}

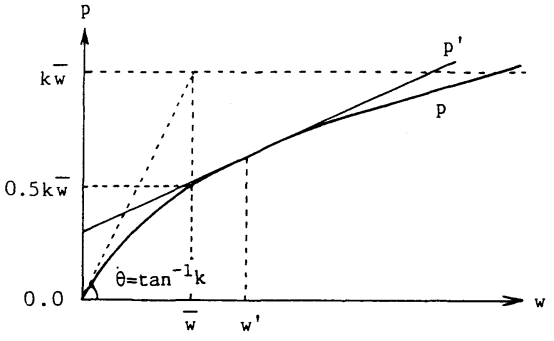

Fig. 6 The reaction-deflection relationship of hyperbolic spring.

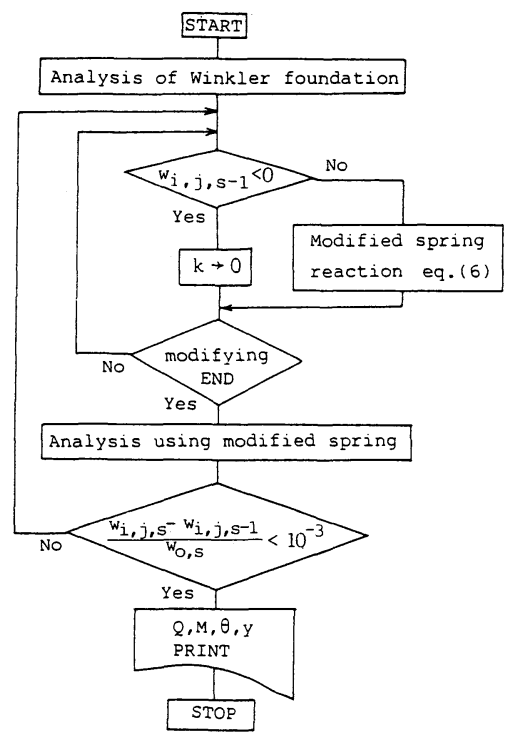

Fig. 7 Flow chart of computational procedures.

$(i, j)$ is expressed $w_{i, j, s-1}$, the $(s)$ th analysis is made as follows.

$$
\begin{aligned}
& w_{i, j, s-1}>0: \text { use the eq. (6) } \\
& w_{i, j, s-1}<0: k=0
\end{aligned}
$$

The process is repeated until convergency condition

$$
\left(w_{i, j, s}-w_{i, j, s-1}\right) / w_{0, s}<10^{-3}
$$

is satisfied. Here, $w_{0, s}$ is the $(s)$ th deflection at the center of the plate.

Rectangular plates of variable thickness which changes linearly in $x$ direction are treated as shown in Fig. 8. The taper ratio is Case (1) $\alpha=0.8, \beta=1.2$, Case (2) $\alpha=0.4, \beta=1.6$, respectively.

The numerical results of rectangular plate of variable thickness on nonlinear elastic foundations are shown together with the numerical results of rectangular plate of uniform thickness on linear (Winkler) and nonlinear elastic foundations in Fig. 9. Fig. 9 shows the values of the bending moments and the deflections at $y=0$. The numerical computation for $m=12$ is carried out. It is found from Fig. 9 that the effects of variable thickness are a little on $M_{y}$, and little on $M_{x}$ and $w$. It is also found that the numerical solutions of uniform thick plate on nonlinear elastic foundations used for the present calculation are about 1.5 and 1.2 times on the deflection and the bending moments at center, respectively, as compared with the solutions on Winkler's foundations. 


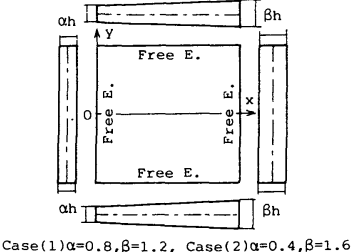

Fig. 8 Rectangular plate with varying thickness.

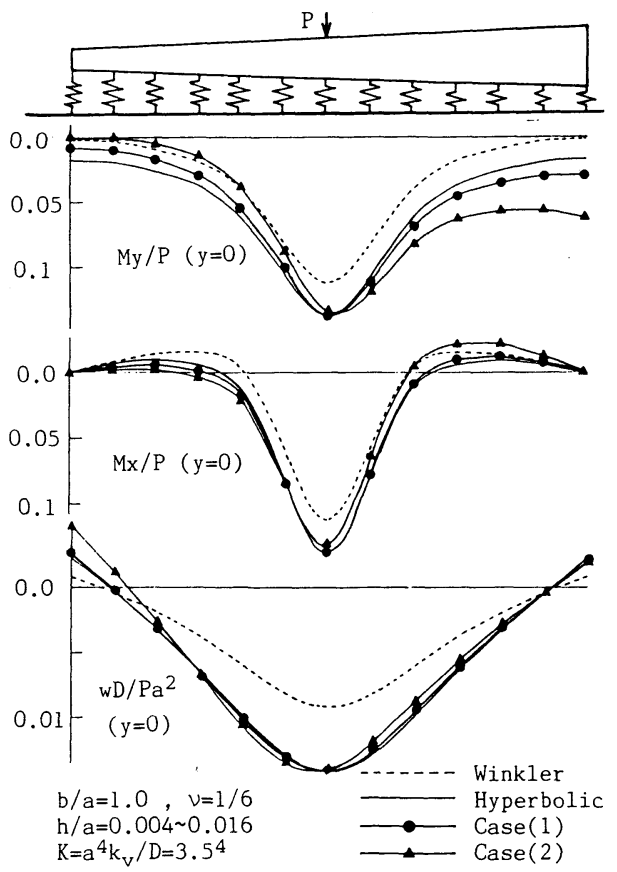

Fig. 9 Numerical solutions of rectangular plate with variable thickness on nonlinear elastic foundations (four free edges).

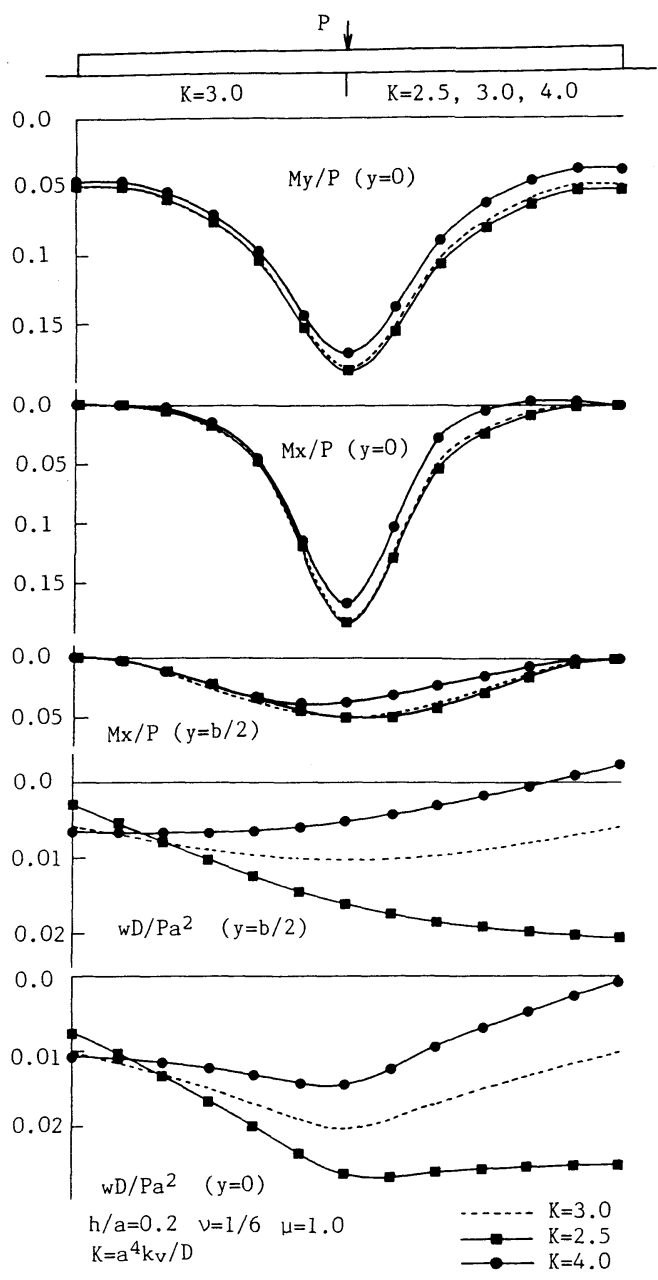

Fig. 10 Numerical solutions of rectangular plate with all free edges on non-uniform elastic foundations.

\section{(3) Rectangular thick plate on non-uniform elastic foundations}

In the previous section, the foundation moduli are uniform in the whole foundations. However, it is considered that the foundation moduli are locally non-uniform.

Accordingly, first let the present method be applied to rectangular plate of uniform thickness with four free edges under a concentrated load $P$ at the center on non-uniform elastic foundations which the values of non-dimensional foundation modulus are $K=3.0$ in left half, $K=2.5,3.0$ or 4.0 in right half as shown in Fig. 10 above. The numerical solutions of the bending moments and the deflections at $y=0$, and $y=b / 2$ are shown in Fig. 10. It is found from this figure that the effects of non-uniformity of dimensionless foundation modulus are very small in regard to the bending moments $M_{y}, M_{x}$, but large in regard to the deflections.

Next, let the present method be applied to rectangular plate of uniform thickness with four free edges, which is subjected to the uniform distributed load $q$ over the square area in the center of plate, on non-uniform elastic foundations as shown in Fig. 11.

Fig. 12 and Fig. 13 show the values of the deflections and the bending moments at $y=0$ and $y=b / 2$. From these figures, it is found that the bending moments are little affected by non-uniformity of foundation moduli, but the deflections are so much. 


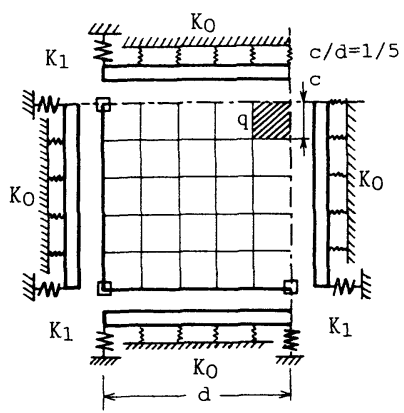

Fig. 11 Rectangular plate on nonuniform foundation.

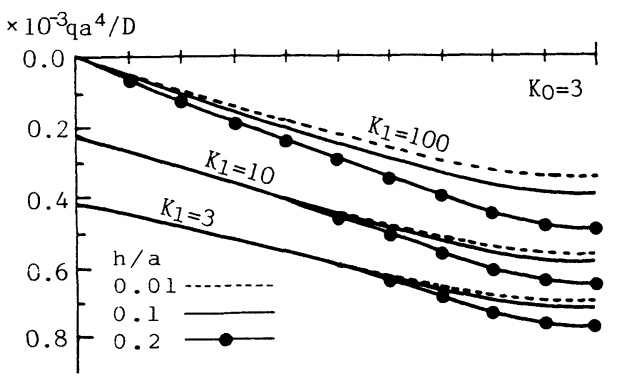

Fig. 12 Deflection curves $(y=0)$ versus $K_{1}$ and $h / a$ values.
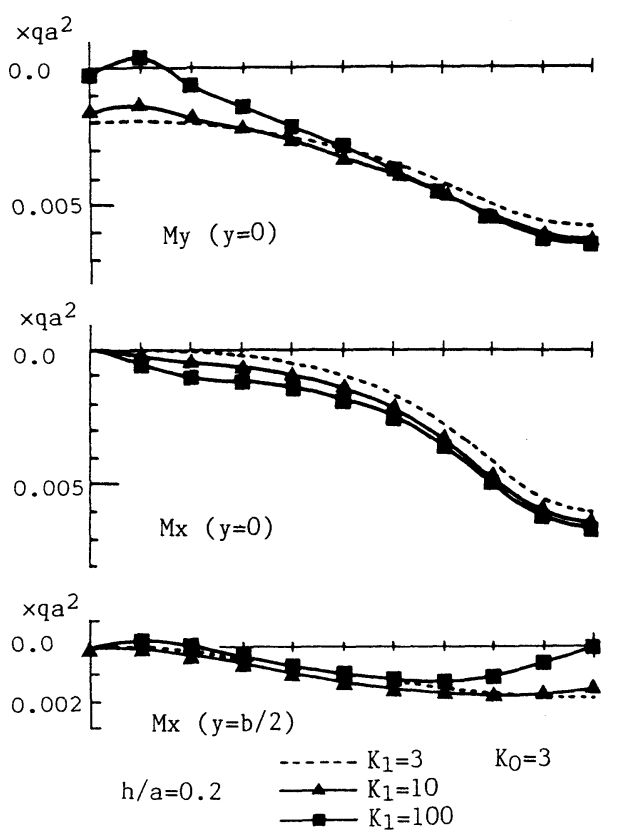

Fig. 13 Bending moments $M_{y}$ and $M_{x}$ versus $K_{1}$ values.

The numerical results for the thin plate $(h / a=0.01)$ are plotted by the dotted lines for comparison in Fig. 12. When dimensionless foundation moduli as shown in Fig. 11 are $K_{1}=3,10$ and 100, the ratios of maximum deflection are $w_{\max }(h / a=0.2) / w_{\max }(h / a=0.01)=1.10,1.15$ and 1.41 , respectively. It is found that the more non-uniformity of foundation modulus increases, the more influence of plate thickness ratio $h / a$ increases.

\section{CONCLUSIONS}

The main conclusions of the work described in this paper can be summarized as follows.

(1) A general numerical method for analyzing the bending behavior of rectangular Mindlin plates on elastic foundations has been proposed. This method is an application of numerical integration and the numerical solution of integral equations.

(2) The approximate solutions of the partial differential equations of the rectangular Mindlin plate give the transverse shear forces, the twisting moments, the bending moments, the rotations and the deflections at the all discrete points which are intersections of the vertical and horizontal equally dividing lines on the plate. Thus, the method does not require prior assumption of the shape of the deflection of the plate.

(3) The method can treat the bending problems of the rectangular plate on nonlinear elastic foundations and on non-uniform elastic foundations with acceptable accuracy and efficiency.

\section{Appendix}

$\begin{array}{llll}A_{p 1}=\gamma_{p 1}, & B_{p 1}=0, & C_{p 1 k l}=\mu\left(\gamma_{p 3}+L_{k l} \gamma_{p 7}\right) & {\left[\gamma_{p t}\right]=\left[\bar{\gamma}_{p t}\right]^{-1}} \\ A_{p 2}=0, & B_{p 2}=\mu \gamma_{p 1}, & C_{p 2 k l}=\mu \gamma_{p 2}+L_{k l} \gamma_{p 8} & \bar{\gamma}_{11}=\beta_{i i}, \bar{\gamma}_{12}=\mu \beta_{j j}, \bar{\gamma}_{22}=-\mu \beta_{i j}, \bar{\gamma}_{18}=\bar{k}_{v i j}, \\ A_{p 3}=\gamma_{p 2}, & B_{p 3}=\mu \gamma_{p 3}, & C_{p 3 k l}=J_{k l} \gamma_{p 6}, & \bar{\gamma}_{23}=\beta_{i i}, \bar{\gamma}_{25}=\mu \beta_{j j}, \bar{\gamma}_{31}=-\mu \beta_{i j}, \bar{\gamma}_{33}=\mu \beta_{j j}, \\ A_{p 4}=\gamma_{p 3} & B_{p 4}=0 & C_{p 4 k l}=I_{k l} \gamma_{p 4}, & \bar{\gamma}_{34}=\beta_{i i}, \bar{\gamma}_{44}=-I_{i j} \beta_{i j}, \bar{\gamma}_{46}=\beta_{i i}, \bar{\gamma}_{47}=\mu \nu \beta_{j j}, \\ A_{p 5}=0, & B_{p 5}=\mu \gamma_{p 2}, & C_{p 5 k l}=I_{k l} \gamma_{p 5} & \bar{\gamma}_{55}=-I_{i j} \beta_{i j}, \bar{\gamma}_{56}=\nu \beta_{i i}, \bar{\gamma}_{57}=\mu \beta_{j j}, \bar{\gamma}_{63}= \\ A_{p 6}=\gamma_{p 4}+\nu \gamma_{p 5}, & B_{p 6}=\mu \gamma_{p 6}, & C_{p 6 k l}=-\mu \gamma_{p 7}, & -J_{i j} \beta_{i j}, \bar{\gamma}_{66}=\mu \beta_{j j}, \bar{\gamma}_{67}=\beta_{i i}, \bar{\gamma}_{11}=-\mu L_{i j} \beta_{i j},\end{array}$




$\begin{array}{llll}A_{p 7}=\gamma_{p 6}, & B_{p 7}=\mu\left(\nu \gamma_{p 4}+\gamma_{p 5}\right) & C_{p 7 k l}=-\gamma_{p 8}, & \bar{\gamma}_{76}=\mu \beta_{i j}, \quad \bar{\gamma}_{78}=\beta_{i l}, \bar{\gamma}_{82}=-L_{i j} \beta_{i j}, \quad \bar{\gamma}_{87}=\beta_{i j}, \\ A_{p 8}=\gamma_{p 7} & B_{p 8}=\gamma_{p 8} & C_{p 8 k l}=\gamma_{p 1} \bar{k}_{v k l} & \bar{\gamma}_{88}=\beta_{j j}, \quad \beta_{i j}=\beta_{i i} \cdot \beta_{j j}\end{array}$

\section{REFERENCES}

1) Nohmachi, S. : On the bending of plates on elastic foundation whose four sides and corners are completely free from any stress, Trans. of JSCE, No. 32, pp. 26 32, 1956 (in Japanese).

2) Kurata, M., Takahashi, H. and Tanihira, T. : On numerical analysis of rectangular plates with all free boundaries on nonlinear foundations, Trans. of JSCE, No. 208, pp. 13 21, 1972 (in Japanese).

3) Kitamura, Y. and Sakurai, S. : The solutions of rectangular plate with four free edges on elastic foundations, Journal of the Japan Society of Civil Engineers, Vol.64-3, pp.61 66, 1979 (in Japanese).

4) Cheung, Y. K. and Zienkiewicz, O.C. : Plates and tanks on elastic foundation-an application of finite element method, Int. Jour. Solids and Structures, Vol. 1, pp. 451 461, 1965.

5) Henry, T., Y. : Flexible plate finite element on elastic foundation, Jour. Structural Division, Proc. ASCE, Vol.96, ST10, pp. 2083 2101, 1970.

6) Svec, O. J. : Thick plates on elastic foundations by finite element method, Jour. Engineering Mechanics Division, Proc. ASCE, Vol. 102, EM3, pp. 461 477, 1976.

7) Sonoda, K. and Kobayashi, H. : Rectangular plates on linear viscoelastic foundation, Jour. Engineering Mechanics Division, Proc. ASCE, Vol. 106, EM2, pp. 323 338, 1980.

8) Kobayashi, H. and Sonoda, K. : Rectangular thick plates on linear viscoelastic foundations, Proc. of JSCE, No. 341, pp. 33 39, 1984.

9) Sakiyama, T. and Matsuda, H. : Bending Analysis of rectangular plates with variable thickness, Proc. of JSCE, No. 338 , pp. 21 $\sim 28,1983$ (in Japanese).

10) Matsuda, H. and Sakiyama, T. : Bending analysis of rectangular plates with variable thickness on nonlinear elastic foundations, Proc. of 9th Symp. on Computational Method in Structural Engineering and related fields, pp. 221 226, 1985 (in Japanese).

11) Salvadri, M. : Numerical computation of buckling loads by finite differences, Transactions of ASCE, Vol. 116, pp. 590 636, 1951.

(Received May 30 1986) 